\title{
Diversity of bats in the Soutpansberg and Blouberg Mountains of northern South Africa: complementarity of acoustic and non-acoustic survey methods
}

\author{
Peter John Taylor ${ }^{1,2,6 *}$, Sandie Sowler ${ }^{3}$, M. Corrie Schoeman ${ }^{2}$ \& Ara Monadjem $^{4,5}$ \\ ${ }^{1}$ School of Environmental Sciences, University of Venda, Private Bag X5050, Thohoyandou, \\ 0950 South Africa \\ ${ }^{2}$ School of Life Sciences, University of KwaZulu-Natal, Durban, South Africa \\ ${ }^{3}$ Mill Cottage, 5 Ampney Crucis, Cirencester, Glos, GL7 5RR, U.K. \\ ${ }^{4}$ All Out Africa Research Unit, Department of Biological Sciences, University of Swaziland, \\ Private Bag 4, Kwaluseni, Swaziland \\ ${ }^{5}$ Mammal Research Institute, Department of Zoology \& Entomology, University of Pretoria, \\ Private Bag 20, Hatfield, Pretoria, 0028 South Africa \\ ${ }^{6}$ Core Team Member, Centre for Invasion Biology, Department of Botany \& Zoology, Stellenbosch University, \\ Private Bag X1, Matieland, 7602 South Africa \\ Received 3 December 2012. Accepted 12 March 2013
}

We surveyed bats at 30 localities in the Soutpansberg and Blouberg Mountains within the newly proclaimed Vhembe Biosphere Reserve (VBR) of northern South Africa, based on ANABAT acoustic recordings (63 nights) conducted in parallel with captures of 260 individuals from harp traps (29 trap-nights) and mist nets (54 trap-nights), and searches of ten day-roosts and two night-roosts. Twenty-four species of bats were captured, or positively identified from roosts, out of 44 species previously recorded for the VBR. For those species captured during the study and one additional commonly recorded species, Chaerephon ansorgei, which was not captured, we compiled a library of ANABAT call parameters for reference calls, based on released bats or bats emerging from known-species roosts. Reference calls were obtained from the study area where possible, or from the closest possible site in the savanna region of southeastern Africa. Using principal component analysis and plots of frequency histograms of selected parameters, we investigated the extent to which reference calls of different species could be distinguished on call parameters. Complete separation was obtained for most species but certain species-pairs or trios showed overlap, particularly amongst molossid bats. Accurate identification of unknown calls was complicated by natural intraspecific variation in echolocation call structure due to habitat and behaviour in our species-rich study area. We advocate a conservative approach whereby species-pairs or groups with overlapping calls are treated as single 'species'. Such underestimation can be partly corrected using rarefaction approaches, as illustrated by data collected from Blouberg Nature Reserve. Particularly when surveying bats in species-rich areas such as the eastern savannas of southern Africa, both acoustic and capture-based surveys are necessary to accurately estimate true species richness. From our capture data and roost searches, we recorded nine to 14 species at four west-east, grouped localities defined by this study. Adding acoustic data using a conservative approach to classify overlapping species-pairs or trios, we obtained minimum richness estimates of 15 to 21 species, values which were close to those predicted by a recent macro-ecological model. We found no evidence for a west-east increase in richness with increasing precipitation as predicted by coarse-scale macroecological predictions.

Key words: ANABAT, Chiroptera, Limpopo Province, South Africa, bat species richness, species diversity.

\section{INTRODUCTION}

A number of hypotheses have been advanced to explain broad geographical (e.g. latitudinal or

\footnotetext{
*To whom correspondence should be addressed
}

E-mail: peter.taylor@univen.ac.za elevational) patterns of variation in species richness, amongst which the productivity hypothesis is often agreed to be one of the most important (Field et al. 2009). However, different predictors may act at different spatial or taxonomic scales and in 


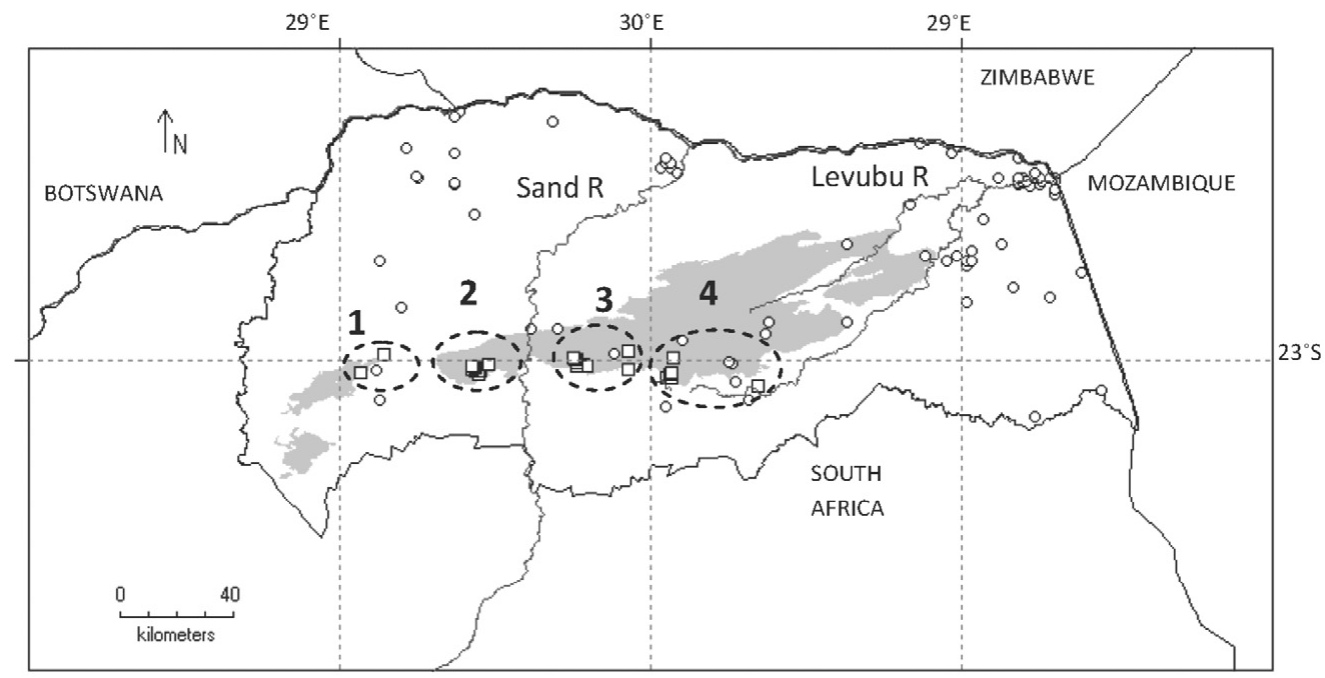

Fig. 1. Map of Vhembe Biosphere Reserve showing locations of sampling sites in the Blouberg and Soutpansberg Mountains (open squares) grouped into four geographical regions (see Table 1), in relation to historical museum collecting localities (open circles) obtained from Monadjem et al. (2010). Grey shading represents extent of the Soutpansberg and the Blouberg Mountains and Mahabeng Plateau.

narrow-ranging versus widespread species, and factors such as spatial autocorrelation and area effects can confound results (Jetz \& Rahbek 2002; Ruggiero \& Kitzberger 2004; Schoeman et al., in press). Macroecological investigations of latitudinal variation in species richness in North American (Patten 2004) and European bats (Ulrich et al. 2007) identified significant latitudinal gradients which could best be explained by climate (temperature and precipitation) and topography. However, these studies identified distinct patterns and predictors in different families of bats. A general climate model was proposed to explain global patterns of elevational richness patterns in bats (McCain 2007), and this model was found to apply to an elevational transect of bats conducted at Mt Mulanje in Malawi (Curran et al. 2012).

In southern Africa, richness of woody plants was shown to be the best predictor of mammalian richness generally (Qian et al. 2009), but in bats of southern and central Africa, habitat heterogeneity (elevational variation) and productivity (climate) are important predictors of species richness (Schoeman et al., in press). The hotspots of greatest species richness were located in the eastern savannas, often associated with mountainous areas such as the Soutpansberg in northern South Africa (Schoeman et al., in press). This confirms the earlier study of Gelderblom et al. (1995) which found the Savanna Biome to be the hotspot of bat species richness in South Africa.
The Soutpansberg Range situated in the Savanna Biome of northern South Africa comprises a recognized Centre for Plant Endemism (Van Wyk \& Smith 2001) with a very rich flora of 3000 plant species and 1066 genera (Hahn 2002). Animal diversity is concordantly high. The Soutpansberg harbours $33 \%$ of South Africa's reptiles, $60 \%$ of its mammals, $75 \%$ of its birds, $50 \%$ of the world's spider families and exceptionally diverse ant communities (Berger et al. 2003; Foord et al. 2008; Munyai \& Foord 2012). Variation in aspect and topography within the Soutpansberg results in strong latitudinal, longitudinal and elevational gradients in climate. Precipitation varies from $367 \mathrm{~mm}$ in the north to $>3000 \mathrm{~mm}$ in the south including mist precipitation (Hahn 2002), making this an ideal study area to corroborate at a local scale, predictions from global hypotheses about species richness (Munyai \& Foord 2012).

The present study reports on a survey of bats in the Soutpansberg and Blouberg Ranges which form the core of the newly proclaimed UNESCO Vhembe Biosphere Reserve (UNESCO 2005). Whilst 44 species of bats have previously been recorded within the boundary of the Biosphere (Monadjem et al. 2010), most were collected from lower altitudes to the east (Kruger National Park) and north (vicinity of Musina) of the Soutpansberg, with almost no collections from the mountains themselves (Fig. 1). The present study included sites from a range of elevations from 600 to $1747 \mathrm{~m}$, 
Table 1. Summary of number of captures (from harp traps and mist nets) and roosts of 24 species of bats grouped into four geographical clusters from west to east in the Blouberg and Soutpansberg (see Fig. 1). \# indicates identifications based on ANABAT calls using reference calls. ? indicates species which can be confused due to call overlap (see text).

\begin{tabular}{|c|c|c|c|c|}
\hline Species & $\begin{array}{c}\text { (1) Blouberg Nature } \\
\text { Reserve: } 845-941 \mathrm{~m} \\
\text { (4 localities) }\end{array}$ & $\begin{array}{l}\text { (2) Luvhondo Nature } \\
\text { Reserve: } \\
956-1747 \mathrm{~m} \\
\text { (11 localities) }\end{array}$ & $\begin{array}{l}\text { (3) Buzzard Mt/Eagles } \\
\text { Nest: } 988-1618 \mathrm{~m} \\
\text { (10 localities) }\end{array}$ & $\begin{array}{c}\text { (4) Piesanghoek/ } \\
\text { Levubu } \\
600-1400 \mathrm{~m} \\
\text { (6 localities) }\end{array}$ \\
\hline Harp trap effort (no. of nights) & 1 & 18 & 6 & 4 \\
\hline Mistnet effort (no. of nights) & 3 & 39 & 10 & 2 \\
\hline $\begin{array}{l}\text { ANABAT recordings } \\
\text { (evenings }-2 \mathrm{~h} / \text { full nights) }\end{array}$ & $0 / 5$ & $0 / 18$ & $7 / 5$ & $16 / 12$ \\
\hline Rousettus aegyptiacus & & & Roost (>100) & \\
\hline Epomophorus wahlbergi & 3 & 6 & Roost (few) & \\
\hline Taphozous mauritianus & $\#$ & Roost (1) & Roost (1-2) \# & $\#$ \\
\hline Nycteris thebaica & Roost (>50) & & & 2 night roosts \# \\
\hline Hipposideros caffer & $\#$ & & & 2 \\
\hline Rhinolophus simulator & $\#$ & $\#$ & Roost (few) \# & $\#$ \\
\hline R. darling & & 3 & 1 & \\
\hline R. clivosus & & $1 \#$ & 5; Roost (few) \# & 1 night roost \# \\
\hline R. smithersi & $1 \#$ & & 1 ; Roost (few) \# & \\
\hline Miniopterus natalensis & 7 \#? & $\# ?$ & $2 \# ?$ & $\# ?$ \\
\hline Pipistrellus hesperidus & $1 \#$ & $90 \#$ & $67 \#$ & $9 \#$ \\
\hline Neoromicia zuluensis & $\#$ & & $2 \#$ & $\#$ \\
\hline Neoromicia capensis & $3 \#$ & $1 \#$ & $\#$ & \# \\
\hline Pipistrellus rusticus & $3 \# ?$ & $\# ?$ & $\# ?$ & $\# ?$ \\
\hline Neoromicia nana & $\#$ & & $\#$ & $1 \#$ \\
\hline Myotis tricolor & \# & & $\#$ & $2 \#$ \\
\hline Myotis welwitschii & $\#$ & $1 \#$ & & $\# ?$ \\
\hline Eptesicus hottentotus & $3 \#$ & $2 \#$ & $5 \#$ & $\#$ \\
\hline Laephotis botswanae & $\#$ & & & $1 \#$ \\
\hline Scotophilus dinganii & 2 \# & 5; Roost $(<10) \#$ & 2; Roost (few) \# & $2 ; 1$ roost \# \\
\hline Chaerephon pumilus & $\# ?$ & \#? & 1; Roost (few) \#? & 1,1 roost \#? \\
\hline Mops condylurus & \#? & $\# ?$ & \#? & $2 ; 2$ roosts $(>10) \# ?$ \\
\hline Mops midas & $\#$ & $\#$ & 2 roosts $(>10) \#$ & 2 roosts $(>10) \#$ \\
\hline Tadarida aegyptiaca & \#? & $1 \# ?$ & \#? & $\# ?$ \\
\hline
\end{tabular}

Not captured;

classified from reference calls

Chaerephon ansorgei \# \#

\# \#? \#?

Unclassified calls

Rhinolophus $\mathrm{Fc}=100 \mathrm{kHz}$

Broad-band FM Fc $42 \mathrm{kHz}$

Broad-band FM Fc $65 \mathrm{kHz}$

Broad-band FM Fc $59 \mathrm{kz}$

Narrow-band Fc $21 \mathrm{kHz}$

Narrow-band Fc $31 \mathrm{kHz}$

Narrow-band Fc $44 \mathrm{kHz}$

Narrow-band FM Fc 57 kHz

\begin{tabular}{|c|c|c|c|c|}
\hline Rhinolophus $\mathrm{Fc}=100 \mathrm{kHz}$ & \# & \# & & \\
\hline Broad-band FM Fc $42 \mathrm{kHz}$ & & & \#? & \#? \\
\hline Broad-band FM Fc 65 kHz & & & & \# \\
\hline Broad-band FM Fc 59 kz & & & \# & \\
\hline Narrow-band Fc 21 kHz & & & $\#$ & \\
\hline Narrow-band Fc 31 kHz & & & & \# \\
\hline Narrow-band Fc 44 kHz & & & & \# \\
\hline Narrow-band FM Fc 57 kHz & & & & $\#$ \\
\hline Total no. of captures & 23 & 110 & 87 & 22 \\
\hline Total no. of species captured & 9 & 10 & 14 & 11 \\
\hline Simpson $D$ & 0.88 & 0.35 & 0.48 & 0.85 \\
\hline ANABAT + captures & 21 & 15 & 20 & 21 \\
\hline No. spp. predicted (model') & 18 & 18 & 27 & 27 \\
\hline
\end{tabular}

${ }^{1}$ Schoeman et al. (in press).

across a west-east rainfall gradient from Blouberg in the west $\left(29^{\circ} \mathrm{E}\right)$ to the Levuvhu Valley in the east $\left(30^{\circ} \mathrm{E}\right)$ along the southern aspect of the mountains. This west-east gradient of increasing rainfall corresponds to a gradient of increasing predicted species richness (Schoeman et al., in press; Table 1). Our data allowed us to sample species richness across this gradient at a local scale so as to make comparisons with predictions based on broad geographic scales. 
Whether testing global hypotheses for species richness, planning core areas for protected areas or assessing environmental impacts of wind farms on bats, it is critical that survey methods adequately and completely sample representative local communities. Our survey concurrently employed multiple approaches using ANABAT bat detectors, a harp trap, mistnets and roost searches. Acoustic surveys have the advantage of being non-invasive and have recently been advocated as the standard method for conducting bat surveys for EIA studies for wind farm proposals in South Africa (Sowler \& Stoffberg 2012). Acoustic surveys depend on the existence of good call reference libraries and may significantly underestimate true species richness, especially in species-rich areas such as savannas. We provide the first ANABAT library of call parameters for reference files for 24 bat species captured during our study in the Soutpansberg and one additional commonly acoustically-recorded species assumed to be Chaerephon ansorgei. We compared and combined data from invasive and non-invasive methods to: a) attempt to sample bat communities as completely as possible, and (b) to assess the performance of ANABAT recordings alone to estimate true species richness of bats in a hotspot of bat diversity. To address the second objective, we investigated the overlap in echolocation call parameters in reference calls of different bat species using multivariate and univariate statistical approaches. We recognize that our results may to some extent be biased by seasonal migration patterns which are known to occur in species such as Myotis tricolor (McDonald et al. 1990), Miniopterus natalensis (van der Merwe 1975) and Eidolon helvum (Richter \& Cumming 2008).

\section{METHODS}

We surveyed 30 localities along the southern aspect of the Blouberg and Soutpansberg Ranges using 63 nights of ANABAT recordings (www.titley.com.au), a two-bank harp trap (Faunatech, Australia) set for 29 nights and 54 mistnet-nights (mistnets supplied by Ecotone, Poland) as well as ad hoc searches for day and night roosts (Table 1). We generally used two 9-m mistnets (of height c. $2.5 \mathrm{~m}$ set at ground level) per night of sampling and sampling was conducted for approximately two hours after sunset or until there was no further bat activity. In narrow flight paths an additional $6 \mathrm{~m}$-long mistnet (of height c. $2.5 \mathrm{~m}$ ) was very occasionally employed. Harp traps were deployed from sunset till sunrise along presumed flightways.

ANABAT S.D. 1 and S.D.2 detectors were used to make recordings of the ultrasonic vocalizations of bats which were stored automatically on an S.D. memory card and later analysed by the ANALOOK programme (Chris Corben, version 0.3.8.13, http://www.hoarybat.com). The ANABAT system is widely used for bat surveys worldwide and has the advantage of being able to record huge volumes of bat call data automatically and efficiently (O'Farrell et al. 1999; Milne et al. 2004; Monadjem et al. 2010; Williams-Guillen \& Perfecto 2011; Skalak et al.2012). In some cases, detectors were deployed continuously from sunset to sunrise whereas in other cases, recording was carried out only for the maximum foraging period of bats, approximately two hours after sunset (Table 1). We noted whether bat detectors were placed in 'open' or 'closed' habitats and where possible attempted to record both open and habitats at each locality surveyed.

All captured bats were sexed and adult status was determined by the completion of ossification of the wing bones. Mass (g) was recorded using a Pesola balance while forearm length $(\mathrm{mm})$ was measured using either vernier or digital callipers. In order to reliably identify each species, voucher specimens (alcohol skins and skulls) were obtained for each species collected in addition to soft tissues (for possible DNA sequencing) and these were deposited in the collection of the Durban Natural Science Museum. Identification was based on matrices in Monadjem et al. (2010) based on external, cranial and dental characters. Collecting was conducted under a permit from the Limpopo Department of Economic Development, Environment and Tourism (Permit No. 001-CPM40300010).

Where possible we obtained ANABAT reference calls of known species from released individuals collected during our study. We also incorporated data from existing libraries of reference calls from the Waterberg, Western Cape, KwaZulu-Natal and Namibia (data supplied by S.S.) as well as from Swaziland, Mozambique and KwaZulu-Natal (data supplied by A.M.).

\section{RESULTS}

A total of 260 individuals of 24 species was captured belonging to all eight southern African families of bats (Table 1). Based on captures, species totals for four grouped localities (Fig. 1) 



Fig. 2. Plots of first two components from principal components analysis for four parameters of reference calls (Fmin, Duration, Fc and Fk) for (a) all vespertilionid/miniopterid bat species, and (b) four species (Neoromicia capensis, Scotophilus dinganii, Eptesicus hottentotus, Laephotis botswanae).

varied from nine (Blouberg Nature Reserve) to 14 (Buzzard Mt/Eagle's Nest). When accounting for species occurrences based on acoustic identifications (as described later), species totals varied from 15 (Luvhondo Private Nature Reserve) to 21 (Blouberg Nature Reserve and Piesanghoek/ Levubu).

Seven acoustic parameters are presented for 25 species based on reference calls obtained from released individuals or individuals recorded emerging from known-species roosts (Table 2). Based on initial PCA's involving these seven parameters, we found four to be useful in separating calls into species within families (three variables were highly variable and obscured species patterns and hence were excluded: slope, Fmax and bandwidth). Variation in reference calls within and between species was summarised for vespertilionid and miniopterid calls using multivariate (Fig. 2) and univariate (Fig. 3) approaches, as was also the case for molossid and emballonurid bats (Figs 4 \& 5). Since calls of rhinolophoid species 
Table 2. Summary statistics for seven acoustic parameters obtained from ANABAT recordings of reference calls from released bats of known identification or emergences from known-species roosts. N1 and N2 refer to number of call sequences (bat passes) and total number of calls, respectively.

\begin{tabular}{|c|c|c|c|c|c|c|c|c|}
\hline Species \& localities & & $\begin{array}{l}\text { Fmax } \\
(\mathrm{kHz})\end{array}$ & $\begin{array}{l}\text { Duration } \\
(\mathrm{m})\end{array}$ & $\begin{array}{l}\text { Bandwidth } \\
\quad(\mathrm{kHz})\end{array}$ & $\begin{array}{l}\mathrm{Fmin} \\
(\mathrm{kHz})\end{array}$ & $\begin{array}{l}\mathrm{Fk} \\
(\mathrm{kHz})\end{array}$ & $\begin{array}{c}\mathrm{Fc} \\
(\mathrm{kHz})\end{array}$ & Slope \\
\hline $\begin{array}{l}\text { FAMILY VESPERTILIONIDAE } \\
\text { Pipistrellus hesperidus } \\
\text { Soutpansberg (Luvhondo, } \\
\text { Buzzard Mt) }\end{array}$ & $\begin{array}{l}\text { Mean } \\
\text { S.D. } \\
\text { N1(N2) } \\
\text { Min } \\
\text { Max }\end{array}$ & $\begin{array}{l}66.9 \\
8.91 \\
19(309) \\
48.5 \\
82.0\end{array}$ & $\begin{array}{c}2.6 \\
0.85 \\
19(309) \\
1.7 \\
5.0\end{array}$ & $\begin{array}{c}22.0 \\
8.24 \\
19(309) \\
5.4 \\
34.8\end{array}$ & $\begin{array}{l}44.9 \\
1.53 \\
19(309) \\
41.3 \\
47.2\end{array}$ & $\begin{array}{l}50.3 \\
2.50 \\
19(309) \\
44.9 \\
54.7\end{array}$ & $\begin{array}{l}45.8 \\
1.78 \\
19(309) \\
41.5 \\
49.1\end{array}$ & $\begin{array}{l}586.0 \\
219.59 \\
19(309) \\
153.8 \\
828.8\end{array}$ \\
\hline $\begin{array}{l}\text { P. rusticus } \\
\text { Waterberg (Lapalala) }\end{array}$ & $\begin{array}{l}\text { Mean } \\
\text { N1 (N2) }\end{array}$ & $\begin{array}{c}62.1 \\
1(7)\end{array}$ & $\begin{array}{l}3.1 \\
1(7)\end{array}$ & $\begin{array}{l}6.8 \\
1(7)\end{array}$ & $\begin{array}{c}55.3 \\
1(7)\end{array}$ & $\begin{array}{c}57.6 \\
1(7)\end{array}$ & $\begin{array}{c}55.4 \\
1(7)\end{array}$ & $\begin{array}{r}294.4 \\
1(7)\end{array}$ \\
\hline $\begin{array}{l}\text { Neoromicia zuluensis } \\
\text { Waterberg (Lapalala) }\end{array}$ & $\begin{array}{l}\text { Mean } \\
\text { S.D. } \\
\text { N1(N2) } \\
\text { Min } \\
\text { Max }\end{array}$ & $\begin{array}{c}73.8 \\
14.93 \\
5(88) \\
59.1 \\
92.8 \\
\end{array}$ & $\begin{array}{l}2.4 \\
0.52 \\
5(88) \\
1.7 \\
3.0 \\
\end{array}$ & $\begin{array}{c}25.7 \\
14.64 \\
5(88) \\
13.4 \\
46.1 \\
\end{array}$ & $\begin{array}{l}48.1 \\
1.94 \\
5(88) \\
45.7 \\
50.5\end{array}$ & $\begin{array}{l}51.3 \\
2.85 \\
5(88) \\
48.4 \\
55.8\end{array}$ & $\begin{array}{l}49.0 \\
1.81 \\
5(88) \\
46.3 \\
51.2\end{array}$ & $\begin{array}{c}666.1 \\
164.55 \\
5(88) \\
487.2 \\
867.5\end{array}$ \\
\hline $\begin{array}{l}\text { N. capensis } \\
\text { Soutpansberg (Luvhondo), } \\
\text { Waterberg (Lapalala), Greyton } \\
\text { (W Cape) }\end{array}$ & $\begin{array}{l}\text { Mean } \\
\text { S.D. } \\
\text { N1(N2) } \\
\text { Min } \\
\text { Max }\end{array}$ & $\begin{array}{l}48.9 \\
6.15 \\
7(215) \\
41.4 \\
61.4\end{array}$ & $\begin{array}{l}3.6 \\
1.05 \\
7(215) \\
2.1 \\
5.0\end{array}$ & $\begin{array}{l}11.4 \\
5.55 \\
7(215) \\
4.7 \\
22.9\end{array}$ & $\begin{array}{l}37.6 \\
1.86 \\
7(215) \\
34.7 \\
40.9\end{array}$ & $\begin{array}{l}40.0 \\
1.92 \\
7(215) \\
37.0 \\
42.4\end{array}$ & $\begin{array}{c}38.2 \\
1.68 \\
7(215) \\
35.8 \\
41.2\end{array}$ & $\begin{array}{c}341.8 \\
88.18 \\
7(215) \\
253.6 \\
509.4\end{array}$ \\
\hline $\begin{array}{l}\text { N. nana } \\
\text { Swaziland (Mlawula), Gillitts } \\
\text { (Durban) }\end{array}$ & $\begin{array}{l}\text { Mean } \\
\text { S.D. } \\
\text { N1(N2) } \\
\text { Min } \\
\text { Max }\end{array}$ & $\begin{array}{l}77.9 \\
3.34 \\
4(74) \\
74.4 \\
80.9\end{array}$ & $\begin{array}{l}3.2 \\
0.59 \\
4(74) \\
2.4 \\
3.6\end{array}$ & $\begin{array}{l}11.7 \\
2.52 \\
4(74) \\
9.5 \\
13.9\end{array}$ & $\begin{array}{c}66.2 \\
0.92 \\
4(74) \\
64.9 \\
67.0\end{array}$ & $\begin{array}{c}68.2 \\
1.60 \\
4(74) \\
66.1 \\
69.9\end{array}$ & $\begin{array}{l}66.9 \\
0.96 \\
4(74) \\
65.4 \\
67.4\end{array}$ & $\begin{array}{c}529.9 \\
220.48 \\
4(74) \\
219.9 \\
733.0\end{array}$ \\
\hline $\begin{array}{l}\text { Scotophilus dinganii } \\
\text { Soutpansberg (Buzzard Mt), } \\
\text { Waterberg (Lapalala) }\end{array}$ & $\begin{array}{l}\text { Mean } \\
\text { S.D. } \\
\text { N1(N2) } \\
\text { Min } \\
\text { Max }\end{array}$ & $\begin{array}{l}51.6 \\
8.19 \\
7(222) \\
42.1 \\
65.2\end{array}$ & $\begin{array}{l}3.4 \\
0.66 \\
7(222) \\
2.7 \\
4.6 \\
\end{array}$ & $\begin{array}{l}18.5 \\
7.85 \\
7(222) \\
9.7 \\
32.0\end{array}$ & $\begin{array}{l}33.1 \\
0.79 \\
7(222) \\
31.6 \\
34.1\end{array}$ & $\begin{array}{l}36.4 \\
1.10 \\
7(222) \\
34.4 \\
37.4\end{array}$ & $\begin{array}{c}33.8 \\
1.03 \\
7(222) \\
31.9 \\
35.3\end{array}$ & $\begin{array}{c}426.1 \\
93.30 \\
7(222) \\
287.1 \\
587.6\end{array}$ \\
\hline $\begin{array}{l}\text { Eptesicus hottentotus } \\
\text { Soutpansberg (Luvhondo) }\end{array}$ & $\begin{array}{l}\text { Mean } \\
\text { S.D. } \\
\text { N1(N2) } \\
\text { Min } \\
\text { Max }\end{array}$ & $\begin{array}{c}60.6 \\
8.23 \\
3(61) \\
54.0 \\
69.8\end{array}$ & $\begin{array}{l}3.1 \\
0.86 \\
3(61) \\
2.6 \\
4.1\end{array}$ & $\begin{array}{c}30.5 \\
8.52 \\
3(61) \\
22.3 \\
39.3\end{array}$ & $\begin{array}{c}30.1 \\
1.74 \\
3(61) \\
28.2 \\
31.6\end{array}$ & $\begin{array}{c}35.2 \\
1.48 \\
3(61) \\
34.2 \\
36.9\end{array}$ & $\begin{array}{c}32.2 \\
1.75 \\
3(61) \\
30.4 \\
33.8\end{array}$ & $\begin{array}{c}553.1 \\
207.87 \\
3(61) \\
359.7 \\
772.9\end{array}$ \\
\hline $\begin{array}{l}\text { Laephotis botswanae } \\
\text { Waterberg (Lapalala) }\end{array}$ & $\begin{array}{l}\text { Mean } \\
\text { N1(N2) } \\
\text { Min } \\
\text { Max }\end{array}$ & $\begin{array}{l}51.6 \\
2(46) \\
50.3 \\
53.0\end{array}$ & $\begin{array}{l}3.0 \\
2(46) \\
2.9 \\
3.1\end{array}$ & $\begin{array}{l}19.6 \\
2(46) \\
18.1 \\
21.1\end{array}$ & $\begin{array}{l}32.0 \\
2(46) \\
31.8 \\
32.2\end{array}$ & $\begin{array}{l}35.6 \\
2(46) \\
35.3 \\
35.8\end{array}$ & $\begin{array}{l}33.0 \\
2(46) \\
32.8 \\
33.3\end{array}$ & $\begin{array}{l}472.5 \\
\quad 2(46) \\
433.8 \\
511.2\end{array}$ \\
\hline $\begin{array}{l}\text { Myotis welwitschii } \\
\text { Soutpansberg (Luvhondo) }\end{array}$ & $\begin{array}{l}\text { Mean } \\
\text { N1(N2) }\end{array}$ & $\begin{array}{c}73.8 \\
1(5) \\
\end{array}$ & $\begin{array}{l}2.1 \\
1(5) \\
\end{array}$ & $\begin{array}{c}40.0 \\
1(5)\end{array}$ & $\begin{array}{c}33.8 \\
1(5) \\
\end{array}$ & $\begin{array}{c}52.9 \\
1(5) \\
\end{array}$ & $\begin{array}{c}50.3 \\
1(5) \\
\end{array}$ & $\begin{array}{r}562.6 \\
1(5) \\
\end{array}$ \\
\hline $\begin{array}{l}\text { M. tricolor } \\
\text { Waterberg (Lapalala), Swaziland }\end{array}$ & $\begin{array}{l}\text { Mean } \\
\text { S.D. } \\
\text { N1(N2) } \\
\text { Min } \\
\text { Max }\end{array}$ & $\begin{array}{l}86.1 \\
5.92 \\
6(120) \\
76.6 \\
91.9\end{array}$ & $\begin{array}{l}2.0 \\
0.40 \\
6(120) \\
1.6 \\
2.6\end{array}$ & $\begin{array}{l}43.3 \\
7.75 \\
6(120) \\
33.6 \\
53.2\end{array}$ & $\begin{array}{l}42.7 \\
4.43 \\
6(120) \\
37.1 \\
50.0\end{array}$ & $\begin{array}{l}57.6 \\
7.25 \\
6(120) \\
48.5 \\
67.5\end{array}$ & $\begin{array}{l}52.4 \\
6.13 \\
6(120) \\
45.3 \\
62.2\end{array}$ & $\begin{array}{c}671.8 \\
359.67 \\
6(120) \\
287.1 \\
1128.9\end{array}$ \\
\hline $\begin{array}{l}\text { FAMILY MINIOPTERIDAE } \\
\text { Miniopterus natalensis } \\
\text { Swaziland, Namibia }\end{array}$ & $\begin{array}{l}\text { Mean } \\
\text { S.D. } \\
\text { N1(N2) } \\
\text { Min } \\
\text { Max }\end{array}$ & $\begin{array}{l}65.9 \\
5.76 \\
21(392) \\
59.0 \\
80.9\end{array}$ & $\begin{array}{c}2.6 \\
0.48 \\
21(392) \\
1.9 \\
3.8\end{array}$ & $\begin{array}{l}12.2 \\
6.00 \\
21(392) \\
5.4 \\
27.4\end{array}$ & $\begin{array}{l}53.7 \\
\quad 0.58 \\
21(392) \\
52.5 \\
54.9\end{array}$ & $\begin{array}{l}56.5 \\
0.31 \\
21(392) \\
55.7 \\
57.0\end{array}$ & $\begin{array}{l}54.1 \\
\quad 0.78 \\
21(392) \\
52.7 \\
55.5\end{array}$ & $\begin{array}{l}379.4 \\
95.78 \\
21(392) \\
235.3 \\
622.8\end{array}$ \\
\hline
\end{tabular}


Table 2 (continued)

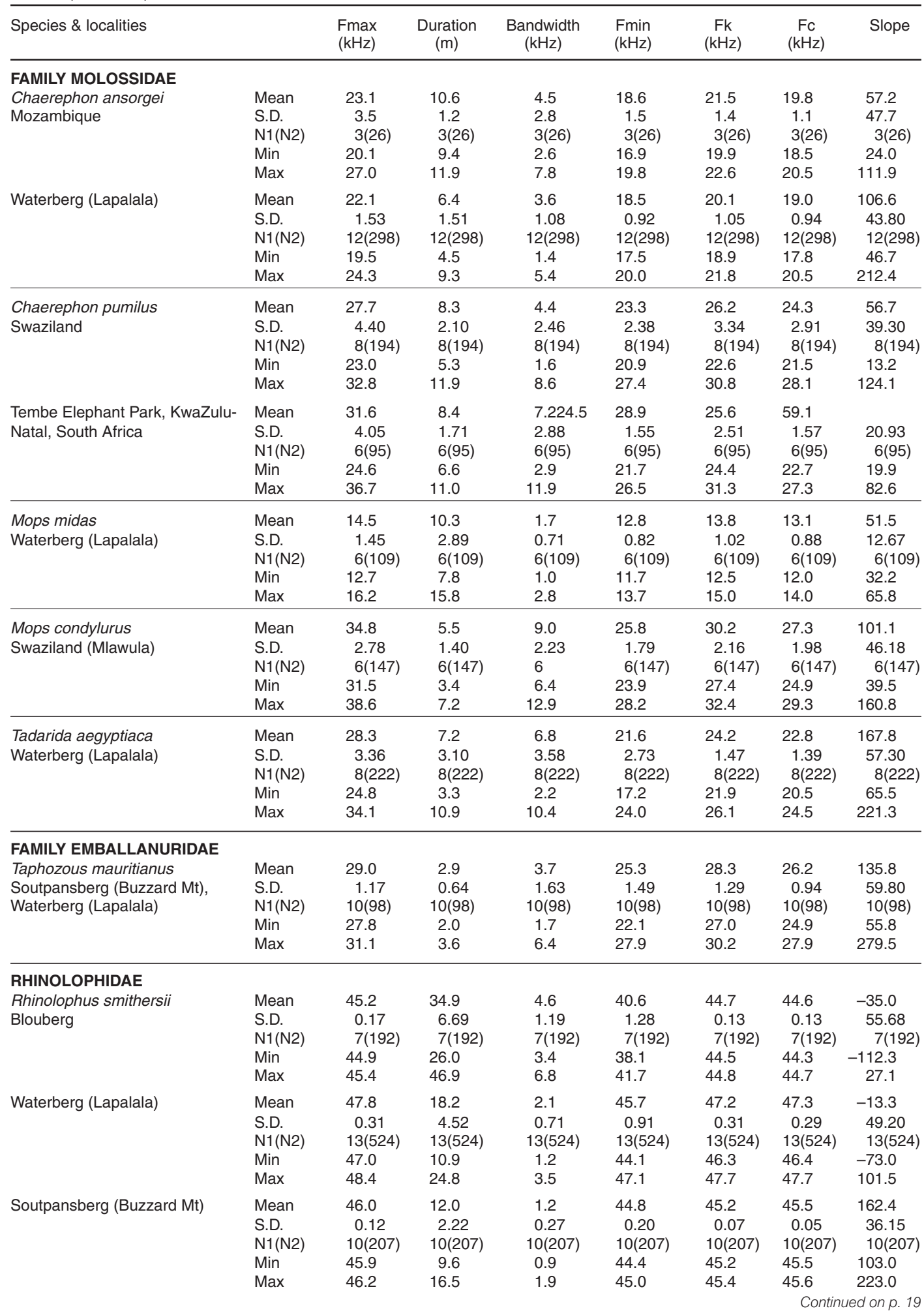


Table 2 (continued)

\begin{tabular}{|c|c|c|c|c|c|c|c|c|}
\hline Species \& localities & & $\begin{array}{l}\text { Fmax } \\
(\mathrm{kHz})\end{array}$ & $\begin{array}{l}\text { Duration } \\
\text { (m) }\end{array}$ & $\begin{array}{l}\text { Bandwidth } \\
\quad(\mathrm{kHz})\end{array}$ & $\begin{array}{l}\text { Fmin } \\
(\mathrm{kHz})\end{array}$ & $\begin{array}{c}\mathrm{Fk} \\
(\mathrm{kHz})\end{array}$ & $\begin{array}{c}\mathrm{Fc} \\
(\mathrm{kHz})\end{array}$ & Slope \\
\hline R. clivosus & Mean & 92.1 & 14.3 & 5.4 & 86.7 & 90.3 & 90.7 & 442.8 \\
\hline \multirow[t]{4}{*}{ Soutpansberg (Buzzard Mt) } & S.D. & 0.51 & 3.63 & 3.46 & 3.21 & 0.42 & 0.40 & 96.80 \\
\hline & N1(N2) & 10(13) & $10(130)$ & 10(130) & $10(130)$ & $10(130)$ & $10(130)$ & $10(130)$ \\
\hline & Min & 91.5 & 8.6 & 2.2 & 79.3 & 89.6 & 90.1 & 280.1 \\
\hline & Max & 92.8 & 19.4 & 13.6 & 89.6 & 90.9 & 91.4 & 605.6 \\
\hline R. darlingi & Mean & 87.4 & 28.0 & 3.2 & 84.2 & 85.6 & 85.1 & 219.8 \\
\hline Waterberg (Lapalala) & N1(N2) & $1(2)$ & $1(2)$ & $1(2)$ & $1(2)$ & $1(2)$ & $1(2)$ & $1(2)$ \\
\hline \multirow[t]{5}{*}{ Swaziland } & Mean & 85.8 & 17.8 & 11.2 & 74.5 & 84.8 & 85.2 & 147.3 \\
\hline & S.D. & 0.25 & 3.39 & 1.77 & 4.54 & 0.52 & 0.54 & 76.28 \\
\hline & N1(N2) & $4(192)$ & $4(192)$ & $4(192)$ & $4(192)$ & $4(192)$ & $4(192)$ & $4(192)$ \\
\hline & Min & 85.5 & 13.3 & 9.5 & 72.3 & 84.1 & 84.5 & 96.8 \\
\hline & Max & 86.0 & 21.5 & 13.7 & 76.1 & 85.2 & 85.6 & 259.2 \\
\hline R. simulator & Mean & 82.0 & 12.4 & 2.7 & 79.3 & 80.5 & 81.5 & 299.3 \\
\hline \multirow[t]{4}{*}{ Waterberg (Lapalala) } & S.D. & 0.58 & 1.71 & 1.35 & 1.42 & 0.46 & 0.47 & 34.63 \\
\hline & N1(N2) & $9(99)$ & $9(99)$ & $9(99)$ & $9(99)$ & $9(99)$ & $9(99)$ & $9(99)$ \\
\hline & Min & 81.1 & 9.6 & 1.8 & 75.9 & 79.8 & 80.9 & 257.3 \\
\hline & Max & 82.9 & 14.4 & 6.0 & 80.6 & 81.2 & 82.1 & 367.6 \\
\hline \multirow[t]{5}{*}{ Swaziland } & Mean & 84.0 & 13.1 & 5.5 & 78.6 & 83.1 & 83.4 & 83.9 \\
\hline & S.D. & 0.34 & 3.66 & 3.63 & 3.77 & 0.83 & 1.20 & 10.28 \\
\hline & N1(N2) & 16.(750) & $16(750)$ & $16(750)$ & $16(750)$ & $16(750)$ & $16(750)$ & $16(750)$ \\
\hline & Min & 83.2 & 7.0 & 1.9 & 71.5 & 80.3 & 79.2 & 69.7 \\
\hline & Max & 84.4 & 18.9 & 12.5 & 82.3 & 83.7 & 84.1 & 101.3 \\
\hline \multicolumn{9}{|l|}{ FAMILY HIPPOSIDERIDAE } \\
\hline Hipposideros caffer & Mean & 142.9 & 4.2 & 19.7 & 123.2 & 141.5 & 142.0 & 181.8 \\
\hline \multirow{4}{*}{ Swaziland } & S.D. & 0.96 & 0.28 & 6.60 & 6.40 & 0.95 & 1.00 & 131.75 \\
\hline & N1(N2) & 13(176) & $13(176)$ & 13(176) & $13(176)$ & 13(176) & 13(176) & $13(176)$ \\
\hline & Min & 140.4 & 3.7 & 12.6 & 108.9 & 139.3 & 139.8 & -133.7 \\
\hline & $\operatorname{Max}$ & 144.1 & 4.7 & 34.1 & 130.3 & 142.9 & 143.5 & 434.8 \\
\hline
\end{tabular}

showed complete non-overlap in frequency parameters (Table 2), no further analysis was necessary. With notable exceptions, based on four acoustic parameters (duration, Fmin, Fk, Fc), PCA grouped 76 recorded reference call sequences into their respective 11 distinct species of vespertilionid and miniopterid bats. Two Laephotis botswanae call sequences grouped either with Scotophilus dinganii or Eptesicus hottentotus. One Pipistrellus rusticus sequence grouped close to sequences of Miniopterus natalensis. Certain species-pairs had calls which, whilst not overlapping, bordered each other closely in multivariate space: Pipistrellus hesperidus/Neoromicia zuluensis; Neoromicia capensis/S. dinganii; and E. hottentotus/S.dinganii). Based on character loadings (Table 3), separation on the first component (PC1) was explained equally by the three frequency parameters. Variation on PC2, which resulted in separation of the two Myotis species from all others, was due to a negative relationship between Fk and Fmin whereby calls of Myotis species had $\mathrm{Fk}$ values much higher than Fmin values compared to other species. This was because in most vespertilionid species, these parameters (Fk and Fmin) were closer in value due to the presence of a 'knee' in their calls. This 'knee' is largely absent in the characteristically broad-band and short-duration calls of Myotis bats. In spite of the general separation of species' reference calls in multivariate space, overlap was detected in reference calls in Fmin (Fig. 3). Thus, minimal overlap was shown between $P$. hesperidus and $N$. zuluensis (Fig. 3a) and between E. hottentotus, $L$. botswanae and S. dinganii (Fig. 3d). Problems of call identification of unknown calls can be further compounded by natural variation, e.g. due to habitat, as shown by frequency histograms of Fmin from recordings of $P$. hesperidus from open and closed habitats (Fig. 3b, c). Despite these problems, analysis of frequency histograms of Fmin from recordings obtained over 63 nights during our survey demonstrated that frequency distributions are often distinctly multi-modal with modes indicative of different species (Fig. 3e-g). Furthermore, careful examination of ANABAT sequences 

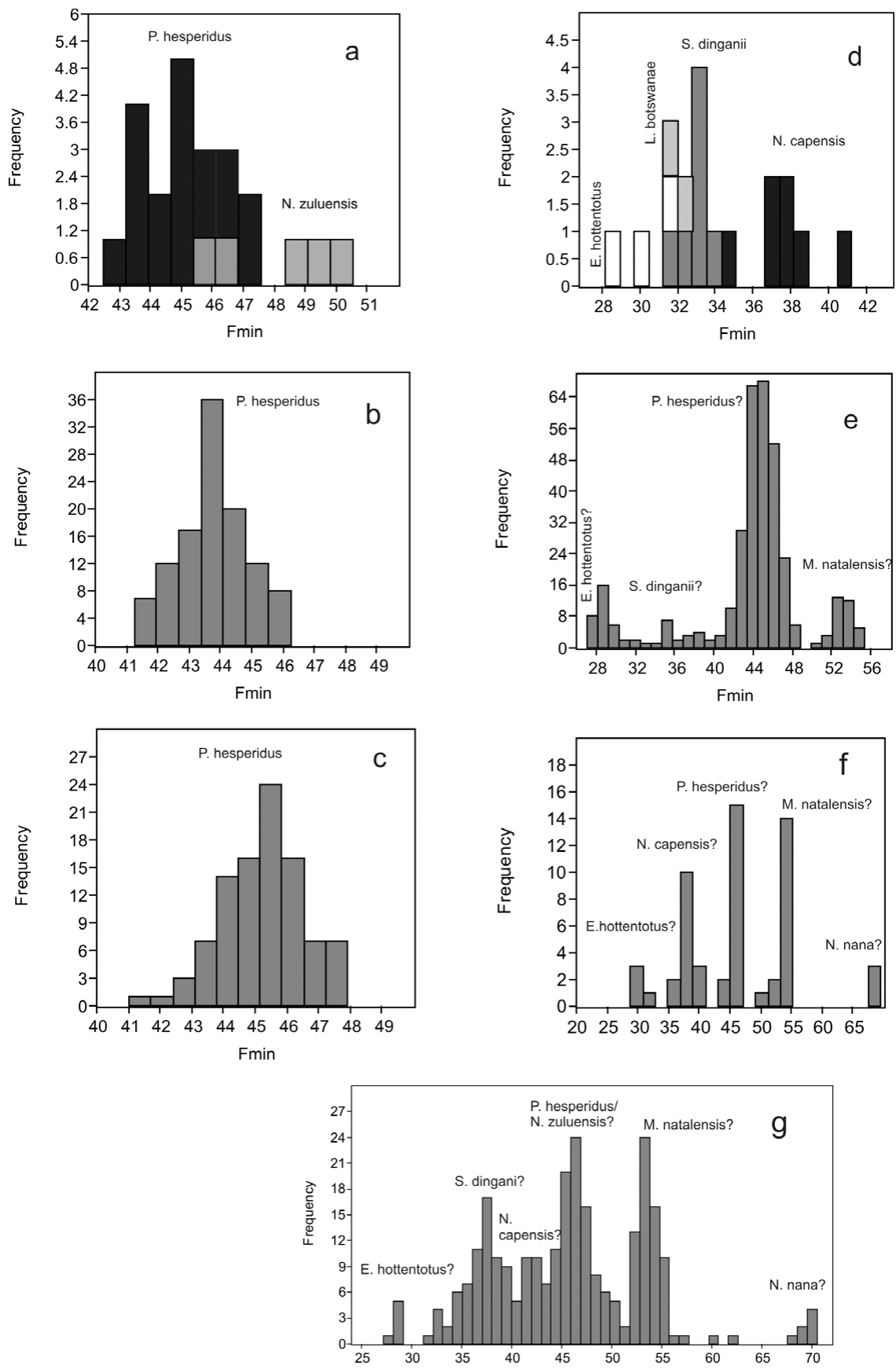

Fig. 3. Frequency histograms for mean Fmin from vespertilionid and miniopterid reference call sequences (a, d) and 'unknown' recordings (b, c, e, f, $\mathbf{g})$. In (a), overlap of Fmin is shown between reference calls of Pipistrellus hesperidus (black shading) and Neoromicia zuluensis (grey shading); (b) and (c) represent mean Fmin for sequences (presumed to be $P$. hesperidus) recorded at the same locality (Lajuma farm house) on consecutive nights in open (forest clearing) and closed (road through forest) habitats; (d) represents reference calls of Neoromicia capensis (black), Scotophilus dinganii (dark grey), Laephotis botswanae (light grey) and Eptesicus hottentotus (white); (e) represents calls recorded from one night at Bergplaats Farm in the far western Soutpansberg; (f) represents calls recorded from one night at Blouberg Nature Reservoir (Mashatu Camp) and (g) represents calls recorded from one night at Blouberg Nature Reserve (Office Reservoir). 


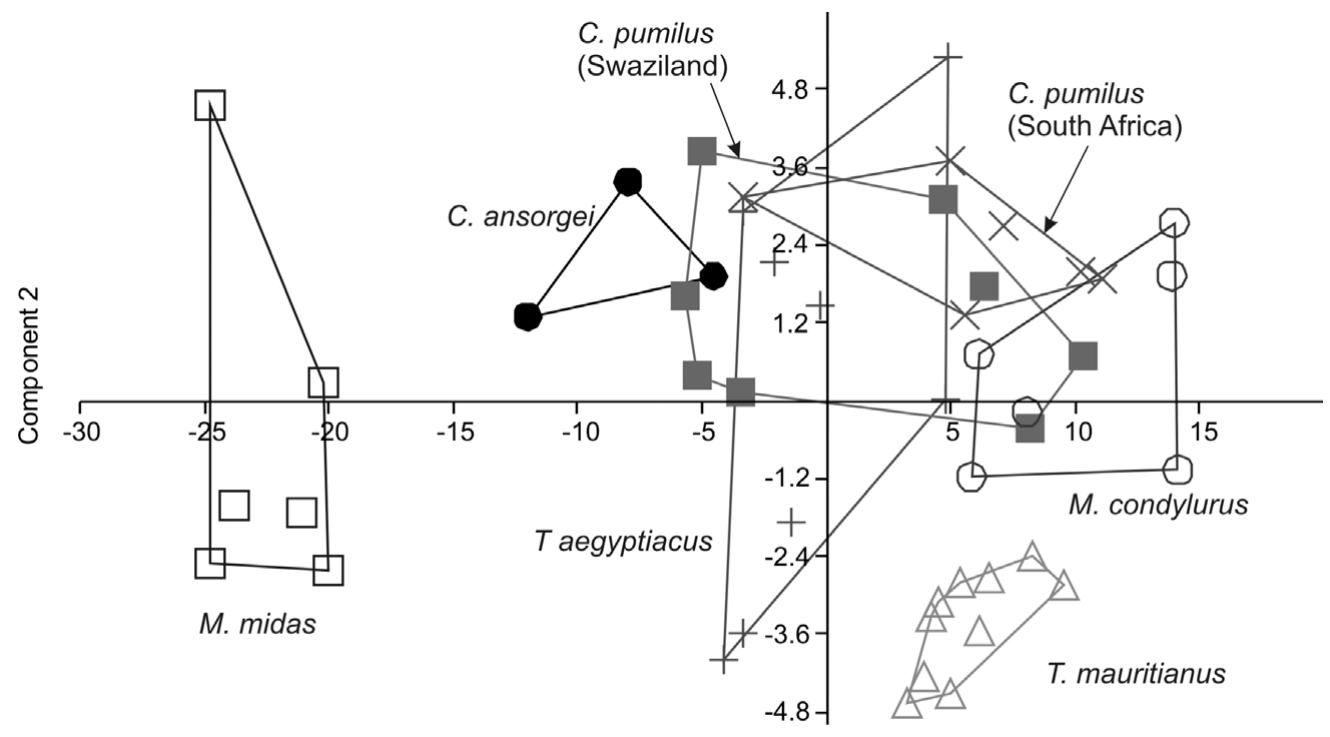

Component 1

Fig. 4. Plot of first two components from principal components analysis for four parameters of reference calls (Fmin, Duration, Fc and Fk) for molossid and emballanurid bats.

from the same habitat where bats of different species having similar calls were recorded flying together assisted in reliable identifications of calls as shown by examples of ANALOOK displays from recordings taken at Blouberg Nature Reserve (Fig. 6).

Of five molossid bats recorded in the study area, reference call sequences of $M$. midas and $C$. ansorgei appear to be distinct whilst those of $C$. pumilus, $T$. aegyptiaca and $M$. condylurus show varying degrees of overlap (Figs 4, 5). As with vespertilionids and minopterids, frequency parameters are the most important variables responsible for species differences (Table 4). In spite of this overlap, recordings of unknown molossids often involved just one or two species and inspection of frequency histograms of Fmin often resulted in clearly multimodal distributions with minimal or no overlap between modes (Fig. 4e); however in some cases, the range of values associated with modes suggested the presence of at least two species (Fig. 4b, c, d, f). Such cases usually involved a combination of $T$. aegyptiaca, $C$. pumilus and M. condylurus.

Using the call reference library (Table 2) and ranges and frequency distributions of key acoustic parameters (e.g. Figs 2, 4), we classified unknown calls obtained during this study (Table 1 ) and used these to update species totals. More detailed analyses of call data are presented elsewhere (Linden et al. 2012; Taylor et al. 2013). We adopted a conservative approach whereby certain species pairs or trios having indistinguishable calls were grouped together. Species richness estimators showed that sampling was, with one exception, incomplete (Table 5; 60-92\%).

Table 3. Component loadings for principal components analysis (PCA) of means of four acoustic variables (Duration, Fmin, Fc and Fk), obtained by ANALOOK from ANABAT sequences from reference calls of 10 species of Vespertilionidae and one of Miniopteridae (see Fig. 2).

\begin{tabular}{lrrrr}
\hline Variable & PC1 & PC2 & PC3 & \multicolumn{1}{c}{ PC4 } \\
\hline Duration (ms) & -0.0165 & 0.0739 & 0.4032 & 0.9120 \\
Fmin (kHz) & 0.5705 & 0.7992 & -0.1869 & 0.0282 \\
Fk (kHz) & 0.5789 & -0.5475 & -0.5305 & 0.2894 \\
Fc (kHz) & 0.5824 & -0.2367 & 0.7218 & -0.2894 \\
\hline
\end{tabular}



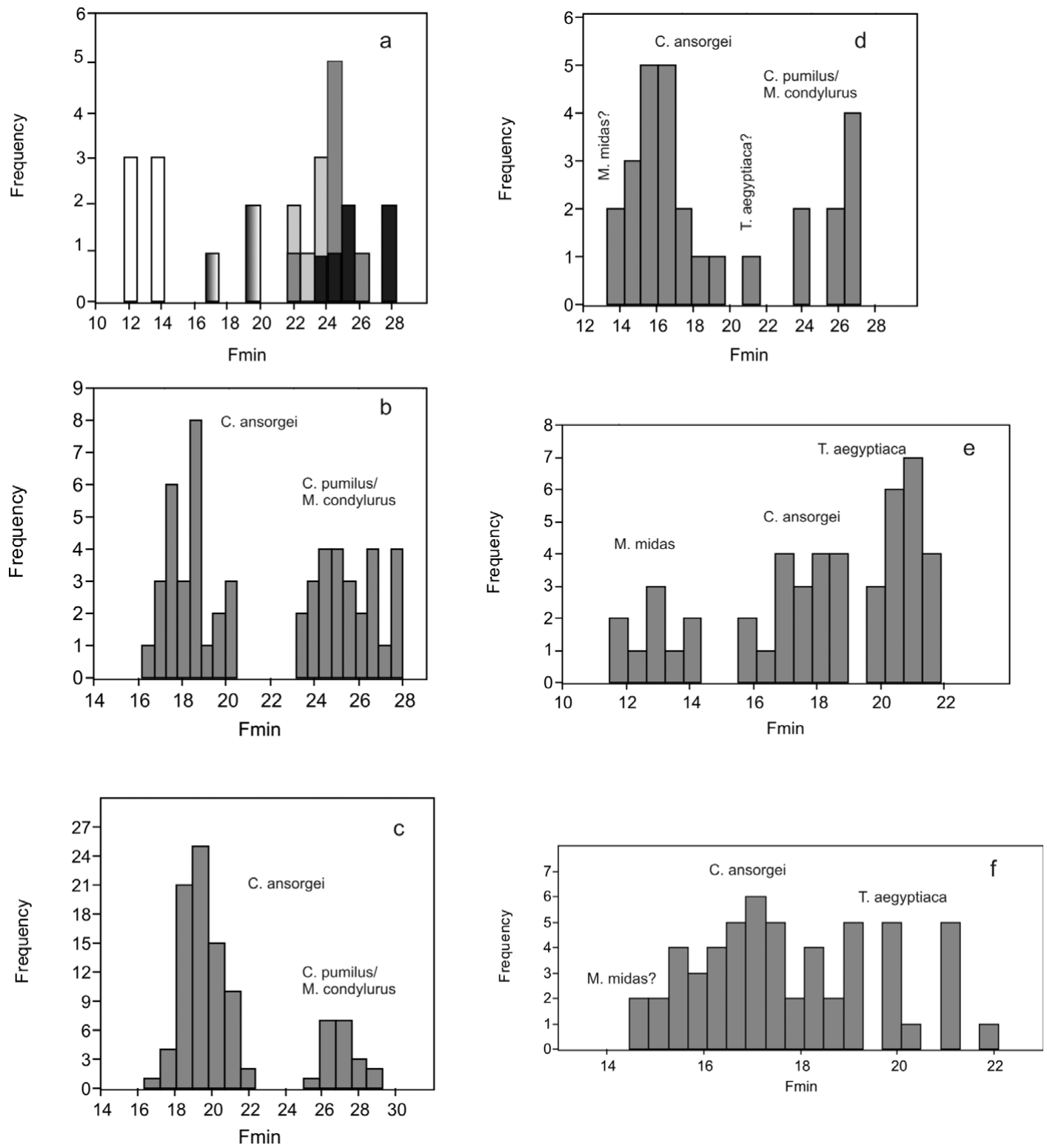

Fig. 5. Frequency histograms for mean Fmin from molossid reference call sequences (a) and 'unknown' recordings (b-f). In (a) distribution of Fmin is shown for reference calls of Mops condylurus (black shading), Chaerephon pumilus (dark grey shading), Tadarida aegyptiaca (pale grey shading), Chaerephon ansorgei (fountain fill shading) and Mops midas (white); distribution of Fmin from recordings of unknown molossid calls shown for selected nights: (b) Blouberg Nature Reserve (Office Reservoir), 18 May 2012; (c) Bergplaats Farm, 25 October 2011; (d) Eagle's Nest Farm, 25 March 2012; (e) Vlakfontein Farm, 21 September 2012.; (f) Buzzard Mt, 7 February 2011.

Richness indicators showed that expected species richness could be as high as 23 species at one site located at the office reservoir. When pooling data for 18 species from four nights from two sites (1290 individual call sequences) located within $500 \mathrm{~m}$ of each other (Mashatu Camp and the office reservoir), richness estimates were between 20 (Chao2) to 24 (Jackknife2) species, suggesting that our sampling was $75 \%$ to $90 \%$ complete. Since we conservatively grouped species with overlapping calls ( $T$. aegyptiaca/C. pumilus/ $M$. condyurus; $M$. natalensis/ $P$. rusticus; and 
a)

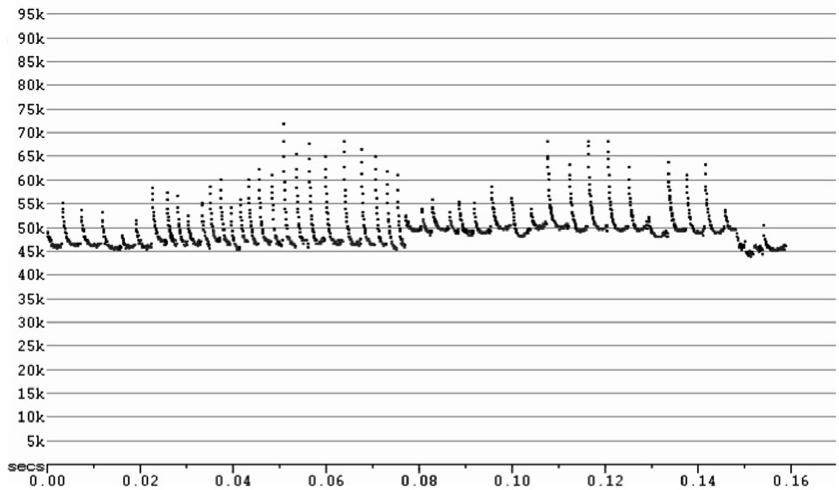

b) 85

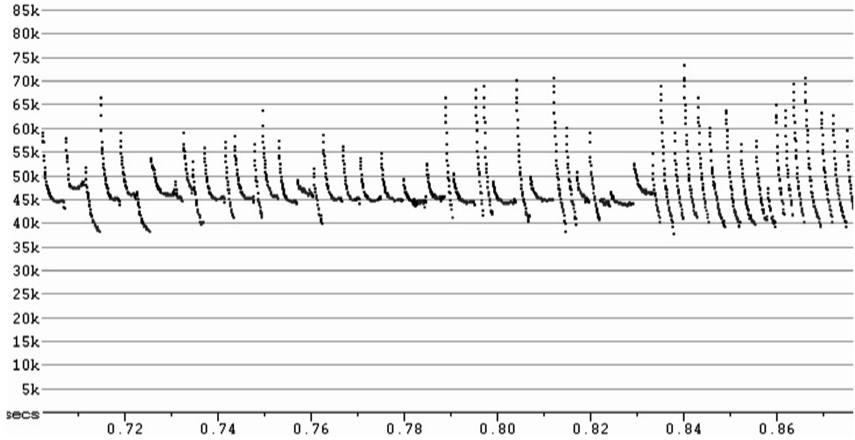

C) $90 \mathrm{k}$



d)

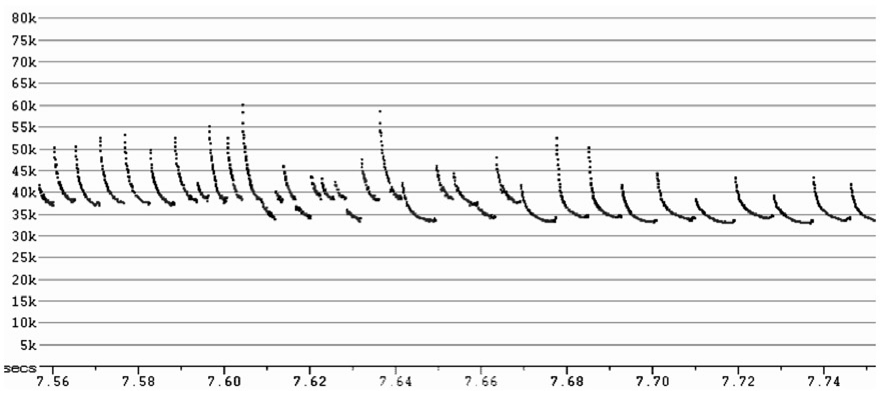

Fig. 6. Selected ANALOOK sonographs recorded on 19 May 2012 from Blouberg Nature Reserve. a, Call sequence showing Pipistrellus hesperidus calls on left and Neoromicia zuluensis on right (spaces between calls removed); b, call sequence showing Myotis tricolor calls overlapping those of $P$. hesperidus (distribution not indicative of feeding buzz); c, call sequence showing feeding buzz within a sequence of Neoromicia capensis calls (distribution of calls indicative of feeding buzz with call duration decreasing and then increasing); although having call parameters similar to $M$. tricolor, the calls in the middle clearly belong to $N$. capensis; d, call sequence showing overlap of calls of N. capensis and Scotophilus dinganii. 
Table 4. Component loadings for principal components analysis (PCA) of means of four acoustic variables (Duration, Fmin, Fc and Fk), obtained by ANALOOK from ANABAT sequences from reference calls of five species of Molossidae and one of Emballanuridae (see Fig. 4).

\begin{tabular}{lrrrr}
\hline Variable (unit) & Axis 1 & Axis 2 & Axis 3 & Axis 4 \\
\hline Duration (ms) & -0.1950 & 0.9772 & -0.0803 & 0.0235 \\
Fmin (kHz) & 0.5166 & 0.1744 & 0.8259 & -0.1437 \\
Fk (kHz) & 0.6245 & 0.0965 & -0.5122 & -0.5816 \\
Fc (kHz) & 0.5524 & 0.0727 & -0.2216 & 0.8003 \\
\hline
\end{tabular}

N. capensis/unidentified vespertilionid with $\mathrm{Fc}$ of $42 \mathrm{kHz}$ ), species richness estimators to some extent compensated for the underestimation caused by our failure to detect at least four 'hidden' acoustic species.

\section{DISCUSSION}

A recent coarse-scale (one-degree square resolution) macroecological model for bat species richness in southern and central Africa predicted local species richness values which were generally close to values obtained independently from local surveys (Schoeman et al., in press). This was corroborated by the current study. The abovementioned model predicts 18 species for the degree-square representing the two westerly localities surveyed in the present study (Blouberg and Luvhondo Nature Reserves) and 27 for the degree-square representing the easterly two (Buzzard Mt/Eagles Nest and Piesanghoek/ Levubu) (Table 1). Given the expected underestimation of species richness using our conservative approach for species identification from acoustic data, our estimated species richness values of 15-21 species were reasonably close to the predicted values. However, we found no evidence for an increase in species richness with precipitation from west to east and the westernmost and easternmost localities had the same richness (21 species). In spite of its much greater sample effort, Luvhondo Private Nature Reserve had the lowest species richness of 15 species as well as the lowest species diversity $(D=0.35)$. The latter is due to domination of the bat community by $P$. hesperidus at higher altitudes $(1300 \mathrm{~m}$ and above); thus based on captures, $P$. hesperidus constituted $82 \%$ of captures at Luvhondo (altitude 956-1745 m), compared to only $4.3 \%$ at Blouberg $(\mathrm{D}=0.88)$ which mostly sampled lower altitudes (845-941 m).

Based only on captures for comparison, we obtained species totals of 9-14 species which matched or exceeded values previously recorded for two protected areas in Limpopo Province, Musina Nature Reserve (eight species) in the Limpopo Valley and Lapalala Wilderness Area (nine species) in the Waterberg Range (Kearney et al. 2008), thus confirming the predictions of the macroecological model of Schoeman et al. (in press) that the Soutpansberg and Blouberg constitute a South African bat richness hotspot.

Our results indicate that neither acoustic (non-invasive) nor capture-based (invasive) methods provide complete estimates of species richness in a bat richness hotspot. A combined approach is essential. Moreover, the extent of overlap in acoustic parameters of certain species reference calls coupled with the extreme intraspecific variability observed due to habitat alone, renders surveys based only on acoustic data as being prone to significant identification error, especially when such surveys (e.g. for wind farm Environmental Impact Assessment studies) are undertaken by consultants who are not bat specialists and in the absence of local reference calls libraries based on captured and released individuals. By providing data on acoustic parameters for 25 savanna bat species, we hope to provide a baseline reference library which can be expanded on by future research. Whilst an automated bat acoustic identification tool has recently been developed for Europe (Walters et al. 2012), this remains a challenging goal in southern Africa, although novel statistical approaches such as that advocated by Agranat (2012) are promising, assuming that a representative selection of reference calls can be compiled.

\section{ACKNOWLEDGEMENTS}

We thank the following for assistance in the field and/or permission to work on their property: Jan Crafford, Hermann De Jager, lan and Retha Gaigher, John and Gail Greaves, Annette Kinealy, Luthendo Liabara, Valerie M. G. Linden, Lappies 
Table 5. Summary of acoustic data for five nights of ANABAT recordings at three sites in Blouberg Nature Reserve during March and May 2012.

\begin{tabular}{|c|c|c|c|c|c|}
\hline Species & $\begin{array}{l}\text { Fountain } \\
19 \text { May }\end{array}$ & $\begin{array}{l}\text { Mashatu } \\
21 \text { March }\end{array}$ & $\begin{array}{c}\text { Mashatu } \\
18 \text { May }\end{array}$ & $\begin{array}{l}\text { Reservoir } \\
18 \text { May }\end{array}$ & $\begin{array}{c}\text { Reservoir } \\
19 \text { May }\end{array}$ \\
\hline \multicolumn{6}{|l|}{ Family Molossidae } \\
\hline Chaerephon ansorgei & 0 & 8 & 11 & 28 & 40 \\
\hline C. pumilus/Tadarida aegyptiaca/Mops condylurus & 0 & 0 & 0 & 1419 & \\
\hline Mops midas & 0 & 0 & 1 & 0 & 0 \\
\hline \multicolumn{6}{|l|}{ Family Hipposideridae } \\
\hline Hipposideros caffer & 0 & 0 & 0 & 1 & 1 \\
\hline \multicolumn{6}{|l|}{ Family Rhinolophidae } \\
\hline Rhinolophus smithersi & 80 & 0 & 2 & 0 & 1 \\
\hline 'Rhinolophus $100 \mathrm{khz}$ & 0 & 0 & 0 & 0 & 5 \\
\hline R. simulator & 17 & 0 & 0 & 0 & 3 \\
\hline \multicolumn{6}{|l|}{ Family Miniopteridae } \\
\hline Minioterus natalensis/Pipistrellus rusticus & 18 & 17 & 7 & 76 & 88 \\
\hline \multicolumn{6}{|l|}{ Family Vespertilionidae } \\
\hline Eptesicus hottentotus & 6 & 3 & 1 & 13 & 10 \\
\hline Laephotis botswanae & 0 & 0 & 0 & 0 & 6 \\
\hline Myotis tricolor & 0 & 0 & 0 & 0 & 18 \\
\hline Myotis welwitchii & 0 & 0 & 0 & 1 & 0 \\
\hline Neoromicia capensis/‘Pip42’' & 9 & 15 & 4 & 84 & 209 \\
\hline Neoromicia nana & 1 & 3 & 0 & 15 & 51 \\
\hline Neoromicia zuluensis & 0 & 0 & 0 & 19 & 103 \\
\hline Pipistrellus hesperidus & 45 & 19 & 7 & 107 & 224 \\
\hline Scotophilus dinganii & 0 & 1 & 0 & 24 & 26 \\
\hline \multicolumn{6}{|l|}{ Family Emballanuridae } \\
\hline Taphozous mauritianus & 0 & 0 & 1 & 2 & 2 \\
\hline No. calls & 176 & 66 & 34 & 384 & 806 \\
\hline No. species & 7 & 7 & 8 & 10 & 16 \\
\hline \multicolumn{6}{|l|}{ Estimated richness } \\
\hline Chao2 & 7.54 & 7 & 12 & 12.55 & 19.03 \\
\hline \% sampling (Chao2) & 92.8 & 100.0 & 66.7 & 79.7 & 84.1 \\
\hline Jack2 & 9.72 & 8.75 & 13.21 & 14.22 & 23.16 \\
\hline \% sampling (Jack2) & 72.0 & 80 & 60.56 & 70.32 & 69.08 \\
\hline \multicolumn{6}{|l|}{ Species diversity } \\
\hline Shannon $S$ & 1.46 & 1.64 & 1.75 & 1.94 & 1.99 \\
\hline Simpson $D$ & 3.43 & 4.81 & 5.39 & 5.61 & 5.44 \\
\hline$D$ (Classic formula) & 0.78 & 0.81 & 0.81 & 0.82 & 0.82 \\
\hline
\end{tabular}

and Kim Labuscagne, Jabu and Birthe Linden, Johnson Madzhuta, Delver Mkhari, Vusani Mphethe, Rhulisani Mthombeni, Tovho Mukwevho, Peter Nemudivhiso, Philip Potgieter, Koos Steyn, Paul, Pierre Thomas and Sina M. Weier,

\section{REFERENCES}

AGRANAT, I. 2012. Bat species identification from zero crossing and full spectrum echolocation calls using HMMs, Fisher Scores, Unsupervised Clustering and Balanced Winnow Pairwise Classifiers. Wildlife Acoustics, Inc. Concord, Massachusetts, U.S.A.

BERGER, K., CRAFFORD, J.E., GAIGHER, I., GAIGHER, M.J., HAHN,N. \& MACDONALD, I. 2003. A first synthesis of the environmental, biological and cultural assets of the Soutpansberg. Leach Printers \& Signs, Louis Trichardt, South Africa. 
CURRAN, M., KOPP, M., BECK, J. \& FAHR, J. 2012. Species diversity of bats along an altitudinal gradient on Mount Mulanje, southern Malawi. J. Trop. Ecol.28: 243-253.

FIELD, R., HAWKINS, B.A., CORNELL, H.V., CURRIE, D.J., DINIZ FILHO, J.A.F., GUE GAN, J-F., KAUFMAN, D.M., KERR, J.T, MITTELBACH, G.G, OBERDORFF, T., O'BRIEN, E.M., \& TURNER, J.R.G. 2009. Spatial species-richness gradients across scales: a meta-analysis. J. Biogeogr. 36: 132-147.

FOORD, S.H., MAFADZA, M., DIPPENAAR-SCHOEMAN, A.S., VAN RENSBURG, B.J.2008. Micro-scale heterogeneity of spiders (Arachnida: Araneae) in the Soutpansberg, South Africa: a comparative survey and inventory in representative habitats. Afr. Zool. 43: 156-174.

GELDERBLOM, C., BRONNER, G., LOMBARD, A. \& TAYLOR, P. J. 1995. Patterns of distribution and current protection status of the Carnivora, Chiroptera and Insectivora in South Africa. S. Afr. J. Zool. 30: 103-114.

HAHN, N. 2002. Endemic flora of the Soutpansberg. University of KwaZulu-Natal, Pietermaritzburg.

JETZ, W. \& RAHBEK, C. 2002. Geographic range size and determinants of avian species richness. Science 297: 1548-1551.

KEARNEY, T., SEAMARK, E.C.J., BOGDANOWIZC, W., ROBERTS, E. \& ROBERTS, A. 2008. Chiroptera of Lapalala Wilderness Area, Limpopo Province, South Africa. Afri. Bat Conserv. News 18: 8-12.

McCAIN, C.M. 2007. Could temperature and water availability drive elevational species richness? A global case study for bats. Global Ecol. Biogeogr. 16: $1-13$.

McDONALD, J.T., RAUTENBACH, I.L., NEL, J.A.J. 1990. Roosting requirements and behaviour of five bat species at De Hoop Guano Cave, southern Cape Province of South Africa. S. Afr. J. Wildl. Res. 20: 157-161.

MILNE, D.J., ARMSTRONG, M., FISHER, A., FLORES, T. \& PAVEY, C.R. 2004. A comparison of three survey methods for collecting bat echolocation calls and species-accumulation rates from nightly Anabat recordings. Wildl. Res. 31: 57-63.

MONADJEM, A., RESIDE, A. \& LUMSDEN, L. 2007. Echolocation calls of rhinolophid and hipposiderid bats in Swaziland. S. Afr. J. Wild. Res. 37(1): 9-15.

MONADJEM, A., TAYLOR, P. J., COTTERILL, F.P.D. \& SCHOEMAN, M.C. 2010. Bats of southern and central Africa: a biogeographic and taxonomic synthesis. Wits University Press, Johannesburg.

MUNYAI, T.C. \& FOORD, S.H. 2012. Ants on a mountain: spatial, environmental and habitat associations along an altitudinal transect in a centre of endemism. J. Insect. Conserv. 16: 677-695.

O'FARRELL, M.J., MILLER, B.W. \& GANNON, W.L.1999. Qualitative identification of free-flying bats using the ANABAT detector. J. Mammal. 80: 11-23.

PATTEN, M.A. 2004. Correlates of species richness in North American bat families. J. Biogeogr. 31: 975985.
QIAN, H., KISSLING, W.D., WANG, X., ANDREWS, P. 2009. Effects of woody plant species richness on mammal species richness in southern Africa. J. Biogeogr. 36: 1685-1697.

RICHTER, H.V., \& CUMMING, G.S. 2008. First application of satellite telemetry to track African straw-coloured fruit bat migration. J. Zool. 275: 172-176.

RUGGIERO, A. \& KITZBERGER, T., 2004. Environmental correlates of mammal species richness in South America: effects of spatial structure, taxonomy and geographic range. Ecography 27: 401-417.

SKALAK, S.L., SHERWIN, R.E. \& BRIGHAM, R.M. 2012. Sampling period, size and duration influence measures of bat species richness from acoustic surveys. Methods Ecol. Evol. 3: 490-502.

SOWLER, S. \& STOFFBERG, S. 2012. South African good practice guidelines for surveying bats in wind farm developments, third draft. Distributed by and produced in cooperation with the Wildlife \& Energy Programme of the Endangered Wildlife Trust, Johannesburg.

SCHOEMAN, M.C., COTTERILL, F.P.D., TAYLOR, P.J. \& MONADJEM, A. In press. Using potential distributions to explore environmental correlates of bat species richness in southern Africa: effects of model selection and taxonomy. Current Zoology 59.

TAYLOR, P.J. 1999. Echolocation calls of twenty southern African bat species. S. Afr. J. Zool. 34: 114-124.

TAYLOR, P. J. 2000. Bats of southern Africa. Guide to biology, identification and conservation. University of Natal Press, Pietermaritzburg.

TAYLOR, P. J. 2005. Order Chiroptera. In: J. D. Skinner \& C.T. Chimimba (Eds), Mammals of the Southern African Subregion (pp. 256-352). Cambridge University Press, Cambridge.

TAYLOR, P. J., MONADJEM, A. \& STEYN, J.N. 2013. Seasonal patterns of habitat use by insectivorous bats in a subtropical African agro-ecosystem dominated by macadamia orchards. African Journal of Ecology, DOI: 10.1111/aje.12066.

ULRICH, W., SACHANOWICZ, K., MICHALAK, M. 2007. Environmental correlates of species richness of European bats (Mammalia: Chiroptera). Acta Chiropterol. 9: 347-360.

VAN WYK, A.E., SMITH, G.F. 2001. Regions of floristic endemism in southern Africa. Umdaus Press, Pretoria.

VAN DER MERWE M. 1975. Preliminary study on the annual movements of the Natal clinging bat. S. Afr. J. Sci. 71: 237-241.

WALTERS, C.L., FREEMAN, R., COLLEN, A., DIETZ, C. FENTON, M.B., JONES, G., OBRIST, M.K., PUECHMAILLE, S.J., SATTLER, T., SIEMERS, B.M., PARSONS, S. \& JONES, K.E. 2012. A continental-scale tool for acoustic identification of European bats. J. Appl. Ecol. 49: 1064-1074.

WILLIAMS-GUILLEN, K. \& PERFECTO, I. 2011. Ensemble composition and activity levels of insectivorous bats in response to management intensification in coffee agroforestry systems. PLOS ONE 6(1): e16502.

Corresponding Editor: M.R. Perrin 